\title{
Experiencia en el manejo de cáncer colorrectal en Méderi-Hospital Universitario Mayor, 2012-2014
}

\author{
Leidy Agudelo ${ }^{1}$, Andrés Isaza-Restrepo ${ }^{1,3}$, Carlos Figueroa², Andrés Monroy², Jorge Padrón², \\ Mariana Villaveces ${ }^{4}$
}

Palabras clave: neoplasias del colon; neoplasias del recto; signos y síntomas; estadificación de neoplasias; epidemiología; complicaciones posoperatorias.

\section{Resumen}

Introducción. El cáncer colorrectal es el tercer cáncer más diagnosticado a nivel mundial en hombres y el segundo en mujeres. En Colombia, es la cuarta causa de muerte por cáncer en ambos sexos. Es importante conocer las características de su presentación clínica en nuestro medio y la experiencia en el manejo de esta neoplasia en instituciones hospitalarias de Colombia que sirvan como referencia para analizar tendencias y resultados.

Materiales y métodos. Se llevó a cabo un estudio de corte transversal de la población con diagnóstico de cáncer

1 Departamento de Cirugía General, Méderi-Hospital Universitario Mayor, Bogotá, D.C., Colombia

2 Departamento de Coloproctología, Méderi-Hospital Universitario Mayor, Bogotá, D.C., Colombia

3 Profesor asociado, Escuela de Medicina y Ciencias de la Salud, Universidad del Rosario, Bogotá, D.C., Colombia

4 Epidemióloga, Universidad del Rosario, Bogotá, D.C., Colombia

Fecha de recibido: 28 de junio de 2017

Fecha de aprobación: 4 de noviembre de 2017

Citar como: Agudelo L, Isaza-Restrepo A, Figueroa C, Monroy A, Padrón J, Villaveces M. Experiencia en el manejo de cáncer colorrectal en Méderi-Hospital Universitario Mayor, 2012-2014. Rev Colomb Cir. 2017;32:262-68. colorrectal atendida entre agosto de 2012 y diciembre de 2014, que corresponde al periodo de funcionamiento del Servicio de Coloproctología de Méderi-Hospital Universitario Mayor.

Resultados. Se atendieron 152 pacientes, $57 \%(n=87)$ eran mujeres. La edad promedio fue de 67 años. Se intervino quirúrgicamente el $91 \%(n=138)$, el $61 \%$ $(n=93)$ por vía laparoscópica y el $25 \%(n=38)$ se encontraron en estadio IV. Solo el 4,6\% $(n=7)$ presentó dehiscencia de la anastomosis. El subtipo histológico más frecuente fue el adenocarcinoma moderadamente diferenciado.

Discusión. Las características demográficas de la serie coinciden con las reportadas en la literatura científica. Como sucede en países similares al nuestro, esta neoplasia se diagnostica en los estadios avanzados, lo que sugiere deficiencia del sistema de tamización y diagnóstico precoz. Los resultados del manejo son similares a los reportados por otros grupos de excelencia.

\section{Introducción}

En los últimos años, la carga de cáncer en el mundo se ha hecho mayor en los países en desarrollo, donde se encuentran hoy alrededor del $57 \%$ de los casos y donde se presenta el $65 \%$ de las muertes por cáncer. Aproximadamente, 14,1 millones de nuevos casos de cáncer y 8,2 millones de muertes ocurrieron en el 2012 en todo 
el mundo ${ }^{1}$. El cáncer colorrectal es el tercer cáncer más diagnosticado a nivel mundial en hombres y el segundo en mujeres, con un estimado de 1,4 millones de casos y casi 700.000 muertes en 2012 .

La disminución de la incidencia del cáncer colorrectal en personas mayores de 50 años en los Estados Unidos, refleja mejores estrategias de tamización que conllevan la resección oportuna de los adenomas precancerosos, mientras que el aumento de la incidencia en los países asiáticos y de Europa oriental parece reflejar la mayor prevalencia de factores de riesgo, como dietas poco saludables, obesidad y tabaquismo ${ }^{1,2}$.

Según la guía de tamización de cáncer colorrectal para Colombia del Instituto Nacional de Cancerología, este es la cuarta causa de muerte por cáncer en ambos sexos. Tal como se mencionó para los países asiáticos, en Colombia ha habido también un aumento constante de la incidencia desde la década de los 80 . Al igual que en el mundo entero, la población más afectada son los mayores de 50 años ${ }^{3}$.

En el presente estudio se describen las características demográficas y clínicas, así como la experiencia en el manejo de los pacientes con cáncer colorrectal en Méderi-Hospital Universitario Mayor, en el periodo comprendido entre agosto de 2012 y diciembre de 2014 .

Méderi-Hospital Universitario Mayor es una institución de cuarto nivel de complejidad y está capacitada para atender la demanda creciente que hay en Bogotá; cuenta con una de las redes hospitalarias más grandes del país: 118 camas de observación, 80 camas de cuidados intensivos y 460 camas para hospitalización. Es un centro hospitalario que promueve el bienestar del individuo, su familia y su entorno en cualquier etapa.

A partir de agosto de 2012, se organizó el Servicio de Coloproctología adscrito al Departamento Quirúrgico, que cuenta con tres cirujanos especializados en Coloproctología y la asistencia permanente de uno o dos fellows y uno o dos residentes de Cirugía General. Desde entonces, el servicio trata la mayoría de los pacientes con enfermedades de colon, recto y ano, por lo que se buscó conocer los resultados de esta primera serie de pacientes con cáncer colorrectal tratados en MéderiHospital Universitario Mayor.

\section{Metodología}

Con el objetivo principal de describir la experiencia en el manejo de las neoplasias malignas de colon y recto en Méderi-Hospital Universitario Mayor, se diseñó un estudio descriptivo retrospectivo de corte transversal de la serie de pacientes con diagnóstico de cáncer colorrectal atendidos en el periodo comprendido entre agosto de 2012 y diciembre de 2014.

Se incluyeron todos los pacientes con diagnóstico histopatológico de cáncer colorrectal que ingresaron por el Servicio de Urgencias, que fueron remitidos de otra institución o que asistieron a la consulta externa, y recibieron atención de los servicios de Coloproctología, Cirugía General u Oncología. Los datos se tomaron en forma retrospectiva de las historias clínicas.

El Servicio de Coloproctología cuenta con una base de datos propia que sirvió para la búsqueda de pacientes con diagnóstico de cáncer colorrectal. Además, se revisó la base de datos del servicio de programación de cirugía y se localizaron los números de las historias clínicas de todos los pacientes registrados en Méderi-Hospital Universitario Mayor por el departamento de sistemas, según los códigos correspondientes del CIE-10. Las diferentes variables fueron tomadas de los registros electrónicos de las historias clínicas institucionales archivadas en el sistema Servinte para luego ser tabuladas y analizadas.

El estudio contó con la autorización del Comité de Investigaciones de Méderi-Hospital Universitario Mayor. Por sus características en cuanto a la revisión documental, sin intervención directa sobre los pacientes y con preservación de su confidencialidad, no requirió consentimiento informado.

Se incluyeron todos los pacientes mayores de 18 años con diagnóstico histopatológico confirmado de cáncer colorrectal. Se excluyeron las pacientes embarazadas y aquellos en quienes se hizo el diagnóstico histológico pero continuaron su tratamiento y seguimiento en otra institución. Todos los pacientes incluidos en el estudio fueron controlados aplicando de manera estricta los criterios de inclusión y de exclusión, previa definición de variables y haciendo la recolección por un único autor en forma consecutiva.

Inicialmente, se analizó la distribución de normalidad de la población en estudio mediante la prueba de Kolmo- 
gorov-Smirnov. El análisis descriptivo de las variables cualitativas se hizo con proporciones y frecuencias, y el de las variables cuantitativas, con medidas de tendencia central, según la población paramétrica o no paramétrica, la media, y los intervalos de confianza del $95 \%$ $\left(\mathrm{IC}_{95 \%}\right) V s$. mediana y valor mínimo y valor máximo. Posteriormente, se calculó la razón de prevalencias del cáncer colorrectal según las diferentes características, con su respectiva significación estadística.

\section{Resultados}

Se incluyeron 152 pacientes con cáncer colorrectal en el periodo de estudio. El $57 \%(\mathrm{n}=87)$ eran mujeres y, el 43 $\%(n=65)$, hombres. El promedio de edad al momento del diagnóstico fue de 67 años (rango: 32-90; $\mathrm{IC}_{95 \%}$ 58-78). La distribución de la población fue normal en todas las variables $(p=0,39)$.

Los principales síntomas y signos motivo de consulta y que llevaron al diagnóstico de la neoplasia, fueron dolor abdominal, sangrado digestivo y pérdida de peso (tabla 1).

El tiempo de evolución de los síntomas al momento del diagnóstico fue de 4 meses, en promedio, con un mínimo de 1 mes y un máximo de 18 meses. De los 152 casos analizados, solo 45 presentaron uno o más de los factores de riesgo descritos en la literatura científica para esta neoplasia, y el tabaquismo fue el más frecuente, seguido por los pólipos en colon (tabla 2).

TABLA 1.

Relación de síntomas o signos al momento del diagnóstico

\begin{tabular}{ll}
\hline Síntoma & $\mathbf{n}$ \\
\hline Perforación intestinal & 0 \\
Deposiciones caprinas & 1 \\
Heces acintadas & 1 \\
Palpación de masa & 8 \\
Obstrucción intestinal & 11 \\
Diarrea & 18 \\
Estreñimiento & 32 \\
Pérdida de peso & 61 \\
Sangrado digestivo bajo & 77 \\
Dolor abdominal & 84 \\
\hline
\end{tabular}

En la tabla 3 se presenta una comparación entre el estadio tumoral al momento del diagnóstico, donde se observa que la mitad de los casos se clasificaron inicialmente en estadio III y, tras la consideración de los hallazgos quirúrgicos y el análisis histopatológico, los estadios tumorales definitivos más frecuentes fueron el IVB y IIIB.

Los procedimientos quirúrgicos practicados y su respectiva frecuencia se presentan en la tabla 4.

La vía de abordaje para los procedimientos fue, en su mayoría, laparoscópica (93 casos, 61,2 \%) versus vía abierta (40 casos, 26,3\%) y la trasanal (4 casos, 2,6\%).

TABLA 2.

Factores de riesgo para cáncer colorrectal en la serie de 152 casos

\begin{tabular}{lc}
\hline Factores de riesgo & n \\
\hline Tabaquismo & 24 \\
Pólipos en colon & 8 \\
Antecedentes familiares de cáncer & 6 \\
Alcohol & 4 \\
Síndromes hereditarios & 3 \\
Obesidad & 0 \\
Colitis ulcerativa & 0 \\
Enfermedad de Crohn & 0 \\
\hline
\end{tabular}

TABLA 3.

Comparación entre el estadio al momento del diagnóstico y el estadio definitivo después del procedimiento quirúrgico, según la clasificación TNM de la Organización Mundial de la Salud

\begin{tabular}{lccccc}
\hline $\begin{array}{c}\text { Estadio } \\
\text { inicial }\end{array}$ & $\mathbf{n}$ & $\mathbf{\%}$ & $\begin{array}{c}\text { Estadio } \\
\text { final }\end{array}$ & $\mathbf{n}$ & $\mathbf{\%}$ \\
\hline I & 8 & 5,3 & I & 14 & 9,2 \\
IIA & 14 & 9,2 & IIA & 10 & 6,6 \\
IIB & 12 & 7,9 & IIB & 16 & 10,5 \\
IIC & 0 & 0 & IIC & 8 & 5,3 \\
& & & IIIA & 2 & 1,3 \\
III & 76 & 50 & IIIB & 27 & 17,8 \\
& & & IIIC & 12 & 7,9 \\
IVA & 8 & 5,2 & IVA & 5 & 3,3 \\
IVB & 32 & 21 & IVB & 38 & 25 \\
No registra & 2 & 1,3 & No registra & 20 & 13 \\
Total & 152 & 100 & Total & 152 & 100 \\
\hline
\end{tabular}


TABLA 4.

Relación de procedimientos quirúrgicos practicados

\begin{tabular}{lcc}
\hline \multicolumn{1}{c}{ Procedimientos } & n & \% \\
\hline Hemicolectomía derecha & 61 & 40,1 \\
Resección anterior de recto & 17 & 11,2 \\
Colostomía derivativa & 17 & 11,2 \\
Proctosigmoidectomía & 16 & 10,5 \\
Hemicolectomía izquierda & 10 & 6,6 \\
Colectomía total & 8 & 5,2 \\
Sigmoidectomía & 7 & 4,6 \\
Resección abdomino-perineal & 6 & 3,9 \\
Ileostomía derivativa & 4 & 2,6 \\
Resección transanal & 4 & 2,6 \\
Proctocolectomía & 1 & 0,6 \\
Tumor irresecable - toma de biopsia & 1 & 0,6 \\
Total general & 152 & 100,0 \\
\hline
\end{tabular}

Se convirtieron a la vía abierta el 9,87 \% (15 pacientes) de los procedimientos abordados inicialmente por vía laparoscópica. Las causas para convertirlos fueron: adherencias, 7,9\% (12 casos); importante distensión de asas, $0,65 \%$ ( 1 caso); tumor adherido al retroperitoneo, $0,65 \%$ (1 caso), y daño en la torre del laparoscopio, $0,65 \%$ (1 caso).

La estancia hospitalaria, en promedio, fue de 8,2 días, con una desviación estándar de 7,2 días, un valor mínimo de 1 día y un valor máximo de 43 días $\left(\mathrm{IC}_{95 \%}\right.$ 4-7 días); la estancia en la unidad de cuidados intensivos tuvo un promedio de 4 días, valor mínimo de 2 días y máximo de 16 días ( $\mathrm{IC}_{95 \%}$ 2-3 días).

En la tabla 5 se presenta la frecuencia de las complicaciones relacionadas directa o indirectamente con el procedimiento quirúrgico.

Los tipos histológicos fueron: adenocarcinoma moderadamente diferenciado, $48 \%$ (73 casos); bien diferenciado, $28 \%$ (43 casos); mal diferenciado, $7 \%$ (11 casos); en $15 \%$ (24 casos) no se encontró el reporte histológico y un caso fue de neoplasia diferente a adenocarcinoma.
TABLA 5.

Relación de complicaciones clínicas de la población en estudio

\begin{tabular}{llcc}
\hline \multicolumn{1}{c}{ Variable } & \multicolumn{1}{c}{ Características } & n & \% \\
\hline Complicaciones & Ninguna & 136 & 89,5 \\
relacionadas & Dehiscencia de anastomosis & 7 & 4,6 \\
con el procedi- & Infección del sitio operatorio & 5 & 3,3 \\
miento & Fístula organizada & 1 & 0,6 \\
& Lesión del hilio renal derecho & 1 & 0,6 \\
& Colección presacra & 1 & 0,6 \\
& Peritonitis & 1 & 0,6 \\
\hline \multirow{2}{*}{ ctras } & Ninguna & 140 & 91,4 \\
& Flebitis & 4 & 2,6 \\
& Tromboembolismo pulmonar & 2 & 1,3 \\
& Infección de las vías urinarias & 2 & 1,3 \\
& Infarto agudo del miocardio & 1 & 0,6 \\
& Enfermedad renal crónica & 1 & 0,6 \\
& agudizada & 1 & 0,6 \\
& Intubación prolongada - & 1 & 0,6 \\
& traqueostomía & & \\
& Neumotórax & & \\
\hline
\end{tabular}

\section{Discusión}

El estudio de la serie de casos atendidos en MéderiHospital Universitario Mayor permitió describir las características de los pacientes y los resultados tempranos de su manejo. La mayoría de los pacientes fueron mujeres y el promedio de edad al momento del diagnóstico fue de 67 años, lo que coincide con lo descrito para estos pacientes en la literatura científica, de la misma forma que con las características de la localización del tumor y los tipos histológicos ${ }^{4}$. En cuanto a la presentación clínica, el síntoma predominante que permitió llegar al diagnóstico fue el dolor abdominal, al igual que lo encontrado en el estudio realizado en el Hospital Universitario de Santander ${ }^{5}$. El tiempo promedio de evolución de los síntomas en esta serie fue de 4 meses. Sin embargo, el estadio era avanzado en más de la mitad de los casos ( $55 \%$ fueron estadios III y IV), lo que permite inferir que la enfermedad tiene un curso subclínico inicial en el que el dolor abdominal de características inespecíficas puede ser interpretado como producto de otras enfermedades, por lo que los pacientes no consultan tempranamente o el dolor es subestimado en las consultas médicas. También, podría representar una dificultad en el acceso a los servicios de salud. 
El análisis de los factores de riesgo evidencia que los más frecuentes en esta serie fueron el tabaquismo y la presencia de pólipos en el colon. Estos últimos son, en general, diagnosticados de manera incidental durante la colonoscopia y, puesto que son considerados los precursores de casi todos los cánceres colorrectales esporádicos ${ }^{6}$, se puede deducir la importancia de este estudio para el diagnóstico precoz del cáncer colorrectal. Sin embargo, su indicación debe responder a una estrategia integral de prevención y tamización. Las personas mayores de 50 años tienen un riesgo del $5 \%$ de por vida para desarrollar cáncer colorrectal, riesgo que aumenta a 10 o $15 \%$ en personas con antecedentes personales de pólipos, enfermedad inflamatoria intestinal o antecedentes familiares de cáncer colorrectal. Es sabido que la progresión de las lesiones premalignas surge de la interacción entre los diferentes factores de riesgo: medioambiental, de la dieta, familiar y hereditario, que se hacen relevantes durante las diferentes etapas de la carcinogénesis colorrectal y sobre las que se debe actuar de forma integral ${ }^{6-8}$.

La clasificación TNM se considera actualmente como el estándar para la estadificación de los pacientes con cáncer colorrectal. Este sistema proporciona la información pronóstica más sólida en esta enfermedad y orienta el tratamiento inicial: neoadyuvancia, cirugía o paliación ${ }^{9}$. En la presente serie, al momento del diagnóstico, el estadio más frecuente fue el III y, tras el estudio histopatológico, el estadio definitivo más frecuente fue el IVB. Esta condición puede ser reflejo de múltiples características del contexto económico, social, cultural y del sector salud. En nuestro país, el número de habitantes ha aumentado, la población pasó a ser predominantemente urbana, con mayor exposición a carcinógenos ambientales, estilos de vida no saludables (tabaquismo, sedentarismo, dieta rica en grasas, carnes rojas) y menor acceso a los servicios de salud, todo lo cual lleva a una mayor incidencia del cáncer colorrectal $\mathrm{y}$ a diagnósticos tardíos ${ }^{10}$.

La variedad de los procedimientos quirúrgicos practicados demuestra la evolución de la cirugía colorrectal actual y la exigencia para el desarrollo de habilidades técnicas cada vez más complejas, con énfasis en las mínimamente invasivas. La cirugía laparoscópica fue considerada por primera vez en 1990 para los pacientes sometidos a colectomía por cáncer. La preocupación inicial se centró en la posibilidad de comprometer la supervivencia a largo plazo debido a resecciones oncológicas incompletas; no obstante, la experiencia ha demostrado que las tasas de recurrencia, supervivencia general, complicaciones, reingresos y reintervenciones son similares a las logradas con la técnica abierta, pero con la ventaja de una recuperación perioperatoria más rápida y un uso más corto de narcóticos parenterales con la cirugía laparoscópica ${ }^{11,12}$. En esta serie, la mayoría de los pacientes $(61 \%, n=93)$ fueron operados por abordaje laparoscópico y la tasa de conversión (10\%, $n=6)$ se encuentra en los rangos reportados en manos de expertos ${ }^{12}$.

La dehiscencia de la anastomosis es una de las complicaciones más frecuentemente descrita después de los procedimientos quirúrgicos del colon. Las diferentes series reportan tasas de entre 1 y $30 \%$, pero los grupos de expertos reportan dehiscencia entre 3 y $6 \%{ }^{13}$, cifras dentro de las que se encuentra esta serie, con 4,6\% $(n=7)$ de los casos. Cabe anotar que el $89 \%$ de los casos fueron atendidos por el Servicio de Coloproctología, subespecialidad relativamente nueva en nuestra institución y que tiene como fin el tratamiento de este tipo de neoplasias de manera integral, ofreciendo mejor calidad de vida y resultados similares a los reportados en la literatura científica en manos de expertos.

En términos de experiencia, según el artículo "Volume and outcome in colorrectal cancer surgery" de Science Direct de 2010, se ha definido como hospital de alto volumen aquellos donde se practican, por cáncer rectal, más de 126 procedimientos anuales, más de 24 cirugías y más de 55 procedimientos combinados. Los hospitales que atienden un alto volumen de pacientes con cáncer tienen una mortalidad posoperatoria significativamente más baja y una mejor supervivencia a largo plazo ${ }^{14} \mathrm{y}$, aunque en nuestra institución no se cumple con esta definición y en este estudio no se cuenta con seguimiento a largo plazo para determinar la supervivencia, sí tenemos una tasa de complicaciones comparable con la de manos de expertos, lo cual disminuye la morbimortalidad.

Cada día es mayor el número de sobrevivientes por cáncer, por lo que se considera importante fortalecer algunos puntos relacionados con el entorno del paciente, como problemas intestinales, disfunción sexual, dolor, estrés sicológico experimentado por el cáncer, y preocupaciones relacionadas con el empleo y la discapacidad, como lo recomienda la guía para la 
detección temprana, diagnóstico, tratamiento, seguimiento y rehabilitación de pacientes con cáncer de colon y recto, del Ministerio de Salud y Protección Social ${ }^{15}$. También, se debe tener en cuenta que juega un papel importante, en los resultados de estos pacientes, el trabajo de un equipo multidisciplinario y la discusión de cada caso en reuniones, como se hace en nuestra institución para obtener mejores resultados.

\section{Conclusiones}

El cáncer colorrectal es una neoplasia con gran impacto a nivel mundial, dado que tiene altas tasas de morbimortalidad porque suele diagnosticarse en estadios avanzados y por el limitado acceso a los servicios de salud; solo se logra tener una buena expectativa de vida si el diagnóstico se hace en los estadios tempranos, razón por la que debemos hacer énfasis en la tamización.

Las complicaciones y sus porcentajes relacionados con el procedimiento quirúrgico en nuestros pacientes, son similares a los reportados en la literatura mundial en manos de expertos, lo cual refleja la importancia de que el manejo y el seguimiento de esta neoplasia estén a cargo de personal con experiencia, como ocurre en nuestra institución.

El cáncer colorrectal es una enfermedad que debe ser manejada por grupos con experiencia, en los cuales se discuta cada paciente en juntas multidisciplinarias para tomar las mejores decisiones en torno a ellos y a sus familiares, en busca de excelentes resultados y mejor calidad de vida.

En nuestro país, se debe trabajar en algunos problemas, como la concentración de la oferta de servicios oncológicos en las principales ciudades del país, la falta de recurso humano especializado, el subdesarrollo de los servicios de cuidados paliativos y de apoyo a las familias y a los cuidadores, y la fragmentación de la prestación de servicios sin articulación entre los servicios preventivos y resolutivos, como lo recomienda el plan nacional para el control de cáncer en Colombia.

\title{
Experience with the management of colorectal cancer at Mederi Major University Hospital, Bogotá, Colombia 2012-2014
}

\begin{abstract}
Introduction: Colorectal cancer is the third most diagnosed cancer in men and the second in women worldwide. In Colombia, it is the fourth leading cause of cancer death in both sexes. It is important to know the characteristics of its clinical presentation in our environment and the experience in the management of this pathology in hospital institutions in Colombia that serve as reference to analyze trends and results.
\end{abstract}

Methods: A cross-sectional study of the population with the diagnosis of colorectal cancer was performed between August 2012 and December 2014, which corresponds to the period of operation of the Department of Coloproctology of Méderi-Hospital Universitario Mayor.

Results: A total of 152 patients were attended, 57\% $(n=87)$ women. The average age was 67 years. $91 \%(n=138)$ were surgically intervened, $61 \%(n=93)$ by laparoscopy and $25 \%(n=38)$ were found in stage IV. Only $4.6 \%(n=7)$ had anastomotic dehiscence. The most frequent histological subtype was moderately differentiated adenocarcinoma.

Discussion: The demographic characteristics of the series coincide with those reported in the literature. As in countries similar to ours, this pathology is diagnosed in advanced stages, suggesting deficiency of screening and early diagnosis in the health system. The results of the management are similar to those reported by other groups of excellence.

Key words: Colonic neoplasms; rectal neoplasms; signs and symptoms; neoplasm staging; epidemiology; postoperative complications. 


\section{Referencias}

1. Torre LA, Bray F, Siegel RL, Ferlay J, Lortet-tieulent J, Jemal A. Global Cancer Statistics, 2012. CA Cancer J. Clin. 2015;65:87108.

2. Siegel R, Naishadham D, Jemal A. Cancer statistics for Hispanics/Latinos, 2012. CA Cancer J Clin. 2012;62:283-98.

3. Guía de práctica clínica explícita basada en la evidencia de tamización de cáncer colorrectal para Colombia. Bogotá: Instituto Nacional de Cancerología; 2011.

4. Naini BV, Odze RD. Advanced precancerous lesions (APL) in the colonic mucosa. Best Pract Res Clin Gastroenterol. 2013;27: 235-56.

5. Ferreira MH. Características clínicas, demográficas e histopatológicas de los pacientes con cáncer colorrectal del Hospital Universitario de Santander. Rev Colomb Cir. 2012;27:213-20.

6. Conteduca V, Sansonno D, Russi S, Dammacco F. Precancerous colorectal lesions. Int J Oncol. 2013;43:973-84.

7. Matsuda T, Kawano H, Hisabe T, Ikematsu H, Kobayashi N, Mizuno K, et al. Current status and future perspectives of endoscopic diagnosis and treatment of diminutive colorectal polyps. Dig Endosc. 2014;26:104-8.

8. Laubert T, Habermann JK, Bader FG, Jungbluth T, Esnaashari $\mathrm{H}$, Bruch HP, et al. Epidemiology, molecular changes, histopathology and diagnosis of colorectal cancer. Eur Surg - Acta Chir Austriaca. 2010;42:252-9.

9. Schneider N, Langner C. Prognostic stratification of colorectal cancer patients: Current perspectives. Cancer Manag Res. 2014; 6:291-300

10. Ministerio de Salud y Protección Social, Instituto Nacional de Cancerología. Plan nacional para el control del cáncer en Co- lombia, 2012-2020. Bogotá: Instituto Nacional de Cancerología; 2012.

11. Fleshman J, Sargent DJ, Green E, Anvari M, Stryker SJ, Beart $\mathrm{RW}$, et al. Laparoscopic colectomy for cancer is not inferior to open surgery based on 5-year data from the COST Study Group Trial. Ann Surg. 2007;246:655-64.

12. Clinical Outcomes of Surgical Therapy Study Group, Nelson H, Sargent DJ, Wieand HS, Fleshman J, Anvari M, Stryker SJ, et al. A comparison of laparoscopically assisted and open colectomy for colon cancer. N Engl J Med. 2004;350:2050-9.

13. Kingham TP, Pachter HL. Colonic anastomotic leak: Risk factors, diagnosis, and treatment. J Am Coll Surg. 2009;208:269-78.

14. van Gijn W, Gooiker GA, Wouters MW, Post PN, Tollenaar RA, van de Velde CJ. Volume and outcome in colorectal cancer surgery. Eur J Surg Oncol. 2010;36(Suppl.1):S55-63.

15. Ministerio de Salud y Protección Social, Departamento Administrativo de Ciencia Tecnología e Innovación en Salud, Colciencias. Guía para la detección temprana, diagnóstico, tratamiento, seguimiento y rehabilitación de pacientes con diagnóstico de cáncer de colon y recto. Bogotá: Ministerio de Salud y Protección Social; 2013. Fecha de consulta: 20 de junio de 2017. Disponible en: http://www.iets.org.co/reportes-iets/Documentacin $\% 20$ Reportes/Gu\%C3\%ADa.Completa.Ca.Colon.y.recto.2013.pdf
Correspondencia: Leidy Agudelo, MD
Correo electrónico: ydie191@hotmail.com
Bogotá, D.C., Colombia 